\section{Praxisorientierte Darstellung!}

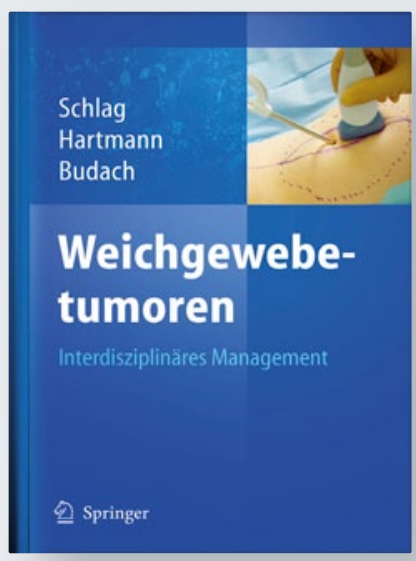

- Interdisziplinärer Leitfaden zu den Diagnoseund Behandlungsverfahren der Weichgewebetumoren

- Einziges deutschsprachiges Fachbuch zum Thema

- Inhaltlich top: von einem namhaften Herausgeber- und Autorenteam

2011.306 S. 190 Abb. Geb.

- $€$ (D) 99,95

$€$ (A) 102,75 | sFr 124,50

ISBN 978-3-642-04938-5

Jetzt bestellen!

\section{Sentinel-Lymphknoten: Bessere Diagnose und Prognose durch SPECT/CT}

Setzt man bei der Suche nach dem Sentinel-Lymphknoten von malignen Melanomen die Kombination aus Einzelphotonen-Emissionscomputertomografie und konventioneller Computertomogafie (SPECT/CT-Verfahren) ein, steigt nicht nur die Trefferquote - auch das krankheitsfreie Überleben der Patienten wird verlängert.

E ür ihre Studie griffen Ingo Stoffels - von der Hautklinik der Essener Universität und seine Kollegen auf die prospektive Melanom-Datenbank des Hautkrebszentrums zurück. Dort fanden sie Angaben zu insgesamt 403 Patienten mit malignem Melanom, die sich zwischen den Jahren 2003 und 2011 bei klinisch negativem Lymphknotenstatus einer Exzision des Sentinel-Lymphknotens unterzogen hatten.

Bei 254 von ihnen war zwischen 2003 und 2008 auf konventionelle Weise mit einer Gamma-Kamera nach diesen Lymphknoten gesucht worden. Nach 2008 stellte die Klinik die Suche auf das SPECT/CT-Verfahren um. Mit dieser Methode können funktionelle und morphologische Bilder fusioniert und auch ein dreidimensionales Szintigramm erstellt werden. Dieser Gruppe waren 149 Patienten zugeordnet. Die Studienteilnehmer beider Gruppen wurden ihren Befunden entsprechend gemäß den Leitlinienvorgaben der Deutschen Dermatologischen Gesellschaft behandelt.

In der SPECT/CT-Gruppe konnten die Ärzte mehr Sentinel-Lymphknoten identifizieren und entfernen - im Durchschnitt 2,40 pro Patient gegenüber 1,87 nach herkömmlichem Suchverfahren ( $\mathrm{p}<0,001)$. Höher lag auch der Anteil der Patienten, bei denen positive Wächter-Lymphknoten gefunden wurden ( 27,5 vs. $18,9 \%$; $\mathrm{p}<0,001)$.

Umgekehrt war die Rate falsch negativer Sentinelknoten - definiert als Rezidiv innerhalb von zwölf Monaten in einer Lymphknotenregion, aus der ein negativer Sentinel-Knoten exzidiert worden war - nach SPECT/CT niedriger: 6,8 gegenüber $23,8 \%(p=0,03)$. Vorteile zeigte das neue Verfahren darüber hinaus bei adipösen Patienten und bei der Lymphknotensuche im Kopf-Hals-Bereich.

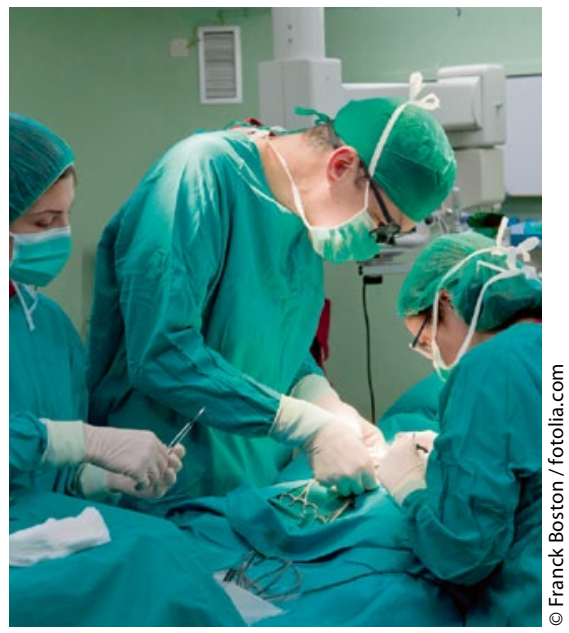

Entfernen von Sentinel-Lymphknoten maligner Melanome - mit SPECT/CT erfolgreicher als mit der Gamma-Kamera.

Der rechnerische Anteil der Patienten, die vier Jahre krankheitsfrei überlebten, erreichte in der SPECT/CT-Gruppe 93,9 im Vergleich zu 79,2\% in der Standardgruppe $(p=0,02)$. Keine signifikanten Unterschiede zeigten sich beim Gesamtüberleben (95,9 bzw. 92,1\%).

Fazit: Die herkömmliche lymphoszintigrafische Suche nach Sentinel-Lymphknoten führt dazu, dass viele der entfernten Knoten tumorfrei sind, obwohl in der Region andere Knoten subklinisch befallen sind. In der Literatur werden dabei Raten von bis zu $44 \%$ genannt. Mit der Kombination SPECT/CT gelingt es, sowohl mehr als auch mehr befallene Wächterknoten aufzuspüren. Das durchschnittliche krankheitsfreie Überleben der Patienten wird dadurch signifikant verlängert.

Robert Bublak

Stoffels I et al. Association between sentinel lymph node excision with or without preoperative SPECT/CT and metastatic node detection and disease-free survival in melanoma. JAMA. 2012;308(10):1007-14. 